\title{
Use of a nerve stimulator to assist cricothyroid membrane puncture during difficult airway topicalization
}

\author{
James S. Green, MBBS • Ban C. H. Tsui, MD
}

Received: 21 May 2015/ Accepted: 28 May 2015/Published online: 3 June 2015

(C) Canadian Anesthesiologists' Society 2015

\section{To the Editor,}

The challenges associated with needle cricothyroidotomy, including identification of the cricothyroid membrane with accurate placement of the needle tip in the tracheal lumen, have been described in a number of articles. ${ }^{1}$ The Fourth National Audit Project of the Royal College of Anaesthetists and the Difficult Airway Society also reported a failure rate of $40-65 \%$ for needle cricothyroidotomy in emergency situations. ${ }^{2}$ Since this procedure is rarely performed, most emergency personnel have either limited or no experience with needle cricothyroidotomy. Factors contributing to high failure rates not only include operator inexperience but also involve the time pressure of an emergency situation. The procedure itself can also be technically challenging, and an additional confirmation tool, such as the use of electrical guidance, ${ }^{3,4}$ could potentially reduce the failure rate. Here, we describe the use of nerve stimulator-guided transtracheal needle placement for topicalization of a difficult airway.

As shown in the Figure and supplementary video (with patient written consent), a cricothyroid membrane puncture is performed using a Stimuplex ${ }^{\circledR}$ HNS12 nerve stimulator (B. Braun, Melsungen, Germany) with a $22 \mathrm{G}$ insulated needle (Pajunk, Geisingen, Germany). Needle puncture of the tracheal wall into the air-filled lumen corresponds with a decrease in current from an initial setting of $0.8 \mathrm{~mA}$ to a minimum current flow (pulse width $0.1 \mathrm{msec}$; frequency $2 \mathrm{~Hz}$ ), an instantaneous audible switch of signal tone, as

Electronic supplementary material The online version of this article (doi:10.1007/s12630-015-0411-2) contains supplementary material, which is available to authorized users.

J. S. Green, MBBS · B. C. H. Tsui, MD ( $\square)$

Department of Anesthesiology and Pain Medicine, University of Alberta, Edmonton, AB, Canada

e-mail: btsui@ualberta.ca well as a change in the visual indicator (green to red) (Figure). When $4 \%$ lidocaine $5 \mathrm{~mL}$ is injected into the trachea, the nerve stimulator shows a corresponding change in current back to the baseline setting of $0.8 \mathrm{~mA}$ with an instantaneous audible switch of signal tone and a change in the colour indicator (red to green). Following transtracheal injection, the flexible bronchoscope is easily passed into the trachea via an oropharyngeal airway.

Transtracheal needle placement is traditionally performed using a needle aspiration technique; however, this can be challenging due to anatomical variation, needle coring, and a considerable learning curve if the procedure is not performed frequently. The technique described here relies on interruption of an electrical circuit to indicate needle tip location. With a grounded electrode on the patient's body, the tip of an insulated needle maintains a closed electrical circuit when in contact with tissue (low impedance). During cricothyroid membrane puncture, the needle tip moves from tissues with low impedance (e.g., skin, subcutaneous tissue, and cricothyroid membrane) into the air-filled tracheal lumen (high impedance), impeding the current and opening the circuit. Conveniently, most modern nerve stimulators have the ability to produce an audible and/or visual signal to warn of an open circuit, enabling easy and rapid detection of the presence or absence (substantially reduced) of an electrical current. As we observed, injection of local anesthetic may again close the circuit, with lidocaine acting as a conducting medium between the needle tip and the inner tracheal wall. Use of an electrical current avoids the worry of needle coring and the need for continuously applying negative pressure on the syringe to confirm intratracheal needle tip placement.

It is important to emphasize that the method described here is limited in its ability to locate the initial needle puncture site, although we have previously described how 
Figure Still images from supplementary video and schematic diagrams show penetration of the tracheal wall by change of electrical current.

(A) Contact with the peritracheal tissue maintains a closed (green light) electrical circuit at an initial setting of $0.8 \mathrm{~mA}$ using a nerve stimulator connected to a $22 \mathrm{G}$ insulated needle. (B) Needle penetration into the tracheal lumen (air medium) impedes the closed circuit to resemble an open circuit (red light) and to signify delivery of a substantially lower current $(0.13 \mathrm{~mA})$. (C) Closed circuit is re-established upon injection of conducting fluid (local anesthetic), with the current returning to baseline level $(0.8 \mathrm{~mA}$ with green light $)$
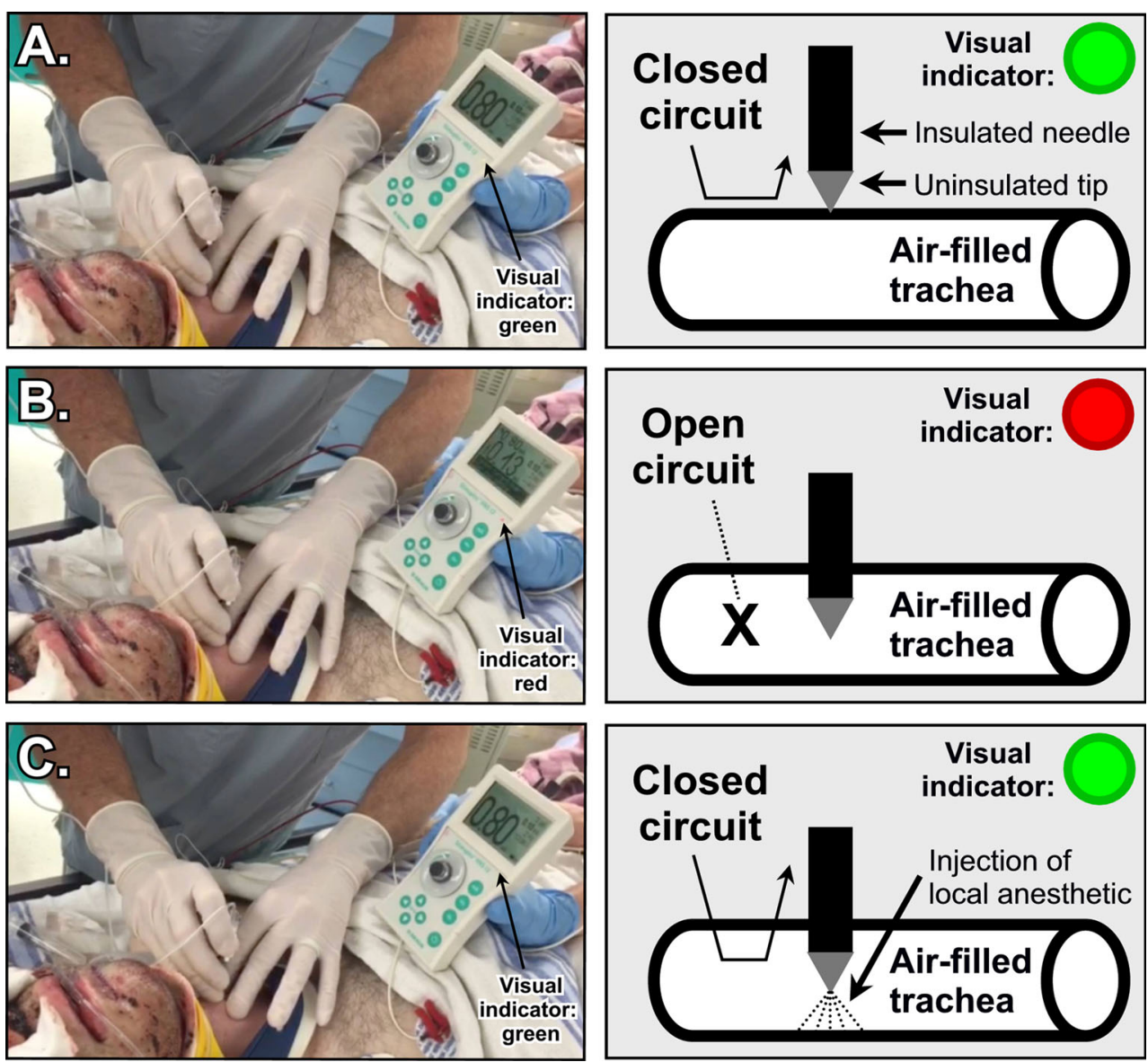

ultrasound imaging can be used to locate both midline and level. ${ }^{5}$ Locating the midline and correct level through careful palpation and/or ultrasound guidance and maintaining the stability of structures during puncture remain crucial components of successful needle placement for both transtracheal techniques. Competence in one technique should be directly transferable to the other; therefore, we anticipate that experience learned from transtracheal injection for airway topicalization may improve success with needle cricothyroidotomy.

Acknowledgements Ban Tsui has a patent licensing agreement with Pajunk for other devices; however, there is no conflict of interest concerning the equipment used in this report. Ban Tsui has been supported by a Clinical Scholar Award from the Alberta Heritage Foundation for Medical Research (AHFMR) and received a CAS/ Abbott Laboratories Career Scientist Award from the Canadian Anesthesiologists' Society.

Conflict of interest None declared.

\section{References}

1. Lamb A, Zhang J, Hung $O$, et al. Accuracy of identifying the cricothyroid membrane by anesthesia trainees and staff in a Canadian institution. Can J Anesth 2015; 62: 495-503.

2. Cook TM, Woodall N, Frerk C; Fourth National Audit Project. Major complications of airway management in the UK: results of the Fourth National Audit Project of the Royal College of Anaesthetists and the Difficult Airway Society. Part 1: anaesthesia. Br J Anaesth 2011; 106: 617-31.

3. Tsui BC, Tsui J. Rapid percutaneous tracheal catheterization using electrical guidance. Can J Anesth 2012; 59: 116-7.

4. Tsui BC, Neufeld A, Walji AH. Comparison of blind and electrically guided tracheal needle insertion in human cadavers. Anaesthesia 2013; 68: 689-93.

5. Green JS, Tsui BC. Applications of ultrasonography in ENT: airway assessment and nerve blockade. Anesthesiol Clin 2010; 28: 541-53. 